\title{
Modified Clark Microsensors with Enhanced Sensing Current
}

\author{
Mehdi Nosrati, Member, IEEE, Daniela Vieira, Edward J. Harvey, Geraldine E. Merle, and Sharmistha Bhadra, \\ Member, IEEE
}

\begin{abstract}
Geometrical modification of three electrode Clark sensor is performed to enhance the sensing amperometric current. Two types of implantable microsensors are designed and characterized for measuring dissolved oxygen $\left(\mathrm{DO}_{2}\right)$. For type I sensor, the area of the reference electrode (RE) conventionally sandwiched between working electrode (WE) and counter electrode (CE), is optimized. In contrast to the conventional Clark sensors, the CE is sandwiched between WE and RE in type II sensor. Both type microsensors are fabricated using simple processes of silicon-based microfabrication technology. Electrochemical characterizations are performed on both sensors. For $96 \% \mathrm{DO}_{2}$, type I and type II microsensors demonstrate a sensing current of $-0.87 \mu \mathrm{A}$ and $-2.35 \mu \mathrm{A}$ as compared to a sensing current of $-0.27 \mu \mathrm{A}$
\end{abstract} for the conventional Clark microsensor of same size. A

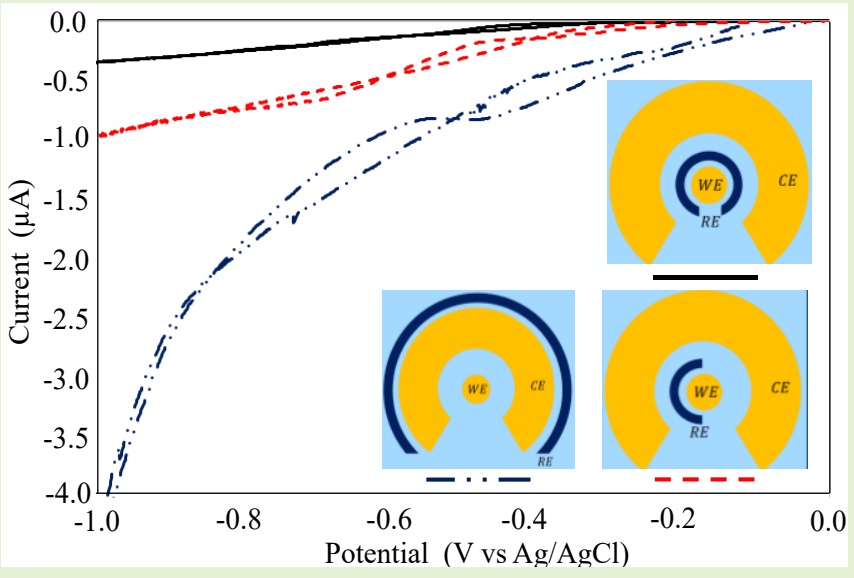
sensitivity of $0.0094 \mu \mathrm{A} / \% \mathrm{DO}_{2}$ and $0.024 \mu \mathrm{A} / \% \mathrm{DO}_{2}$ are achieved with type I and type II microsensor, respectively in contrast to a sensitivity of $0.0027 \mu \mathrm{A} / \% \mathrm{DO}_{2}$ of the conventional Clark microsensor of same size. The sensors show a lifetime of $\mathbf{4 8}$ hours. With its miniature size and enhanced sensing current the sensors have potentials for clinical and biomedical applications.

Index Terms- Dissolved oxygen ( $\left.\mathrm{DO}_{2}\right)$, Clark micro-sensor, capacitive cross-coupling, microfabrication, sensing current, stability.

\section{INTRODUCTION}

$\mathrm{E}$ lectrochemical sensors monitor and correlate an electrochemical reaction to a measurable signal [1], [2]. They are low cost and easy to use, and have low response time. They are widely used in clinical analysis, environmental monitoring, and the food industry. Dissolved Oxygen $\left(\mathrm{DO}_{2}\right)$ is one of the important chemical parameters measured with electrochemical sensors. It is an important parameter in assessing water quality as it influences the organisms living within a water body. In a waterbody, $\mathrm{DO}_{2}$ is an essential factor second only to water itself. $\mathrm{A} \mathrm{DO}_{2}$ level that is too high or too low can harm aquatic life and affect water quality [3].

In medicine, traumatic brain injury (TBI) is a leading cause of morbidity and mortality, especially among those under 45 years of age. In addition to the initial mechanically-induced brain tissue injury, the lack of a sufficient oxygen supply to brain tissue is considered a major cause for the development of secondary brain damage. Studies have shown that brain

Manuscript received XXXX. Mehdi Nosrati and Sharmistha Bhadra are with the Department of Electrical and Computer Engineering, Daniela Vieira, Edward J. Harvey, and Geraldine E. Merle are with Department of Surgery, McGill University, Montreal, QCH3A0E9, (e-mail: mnosrati@ualberta.ca;sharmistha.bhadra@mcgill.ca;daniela.vieira@mail.mcg ill.ca; edward.harvey@mcgill.ca; geraldine.merle@mcgill.ca). tissue oxygenation closely correlates with several outcome parameters and patient prognosis. Electrochemical sensors that are capable of measuring the oxygenation of damaged brain tissue or tissue at risk, and offer extended monitoring options in these patients, are highly desirable in medical applications [5].

The primary platform for electrochemical sensors is Clark amperometric three-electrode structure. Commercially available electrochemical oxygen sensors are large and not biocompatible. Therefore, they are not suitable for in-vivo clinical and biomedical applications [5]. With the recent advancements in microelectromechanical (MEMS) technology and materials, the rapid development of microsensors is reported for clinical and biomedical applications such as dissolved glucose, lactate, glutamine, urea, cholesterol, food pathogenic bacteria, and dissolved oxygen sensors [2]-[10]. A Clark sensor typically consists of three concentric circularshaped electrodes: working (WE), reference (RE) and counter (CE) electrodes [11]. Durability and reliability of electrodes, sufficient sensitivity to measurement species, minimum reaction to surroundings and a measurable output signal (current between working and counter electrodes) are the challenges to be addressed for the realization of a miniaturized Clark electrochemical sensor [12]-[17]. Although miniaturized Clark sensors are desirable for in-vivo clinical and biomedical 
applications, their sensing amperomtric current is small [18, 1]. Noise and small sensing current reduces the reliability and accuracy of the measurement done by Clark microsensors [11].

In order to increase the sensing current of Clark $\mathrm{DO}_{2}$ microsensor, several techniques have been proposed. In [19] the sensing current of a printed circuit board based Clark $\mathrm{DO}_{2}$ microsensor has been increased by increasing the size of the WE. However, the sensor is too large to be used for in-vivo applications. A Clark $\mathrm{DO}_{2}$ microsensor has been fabricated using low temperature co-fired ceramic (LTCC) electronic packaging materials in [20]. The application of LTCC electronic packaging materials has provided high density and long term stability of the reference electrode resulting in enhanced stable sensing current. In [5] a freestanding miniaturized Clark oxygen sensor has been reported. In this work an electrode fabrication scheme incorporating partial adhesion layer and a mesh electrode morphology has been developed to enhance the sensing current. Although the size of the sensors in [5] and [20] are suitable for in-vivo application, their fabrication process is more complicated than conventional Clark microsensors.

Symmetric circular-shaped electrodes are reported to be the optimized geometry for the sensor due to diffusional mass transportation of electrochemical species in all radial directions [11], [5]. Several electrode design are considered to improve device response and sensor stability of Clark sensors. The sensing current of Clark sensor can be increased by a sufficient working electrode area to support oxygen reduction. However, the sensing current is proportional to working electrode area upto a certain size of the electrode and after that it saturates. A high surface area ratio between the counter and working electrodes is required to remove the oxidation of the electrode material. A sufficient reference electrode area is also required to maintain a stable reference voltage. Additionally, the performance of the sensor is correlated to the relative positions as well as the distance between each pair of the electrodes [5].

In addition to the design considerations discussed above, the realization of the two static capacitive coupling between WE$\mathrm{RE}$ and WE-CE is another main consideration in the optimized performance of the sensor. An increase in the capacitive coupling between WE and CE can enhance the sensing current and increase sensitivity. This consideration is associated with the geometrical configuration of the electrodes in a sensor. In this work we aim to enhance the sensing amperometric current between WE and CE and sensitivity of the sensor by geometrically modifying the area and position of the reference electrode of a conventional Clark $\mathrm{DO}_{2}$ sensor. We reported two types of geometrically modified sensors.

In a conventional three-electrode Clark sensor, the RE is sandwiched between WE and CE (see Fig. 1). It is shown that a ratio of static capacitive coupling between the $\mathrm{WE}$ and $\mathrm{CE}$ is coupled to the RE due to diffusional mass transportation of electrochemical species over the RE and it results in an unwanted capacitive coupling between the RE and CE. In type I sensor, geometric area of the reference electrode is optimized to decrease the unwanted capacitive coupling between CE-RE. In contrast to the conventional structure, the $\mathrm{CE}$ is sandwiched between WE and RE in type II microsensor. In this case, a ratio of static capacitive coupling between the WE and RE is coupled to the $\mathrm{CE}$ due to diffusional mass transportation of electrochemical species over the CE. This extra coupling is shown to enhance capacitive coupling between WE-CE and hence the output current between WE and CE. Both geometrically modified structures are microfabricated on silicon wafer. Cyclic voltammetry and chronoamperometry results show that both of them have higher output current and higher sensitivity than the conventional Clark sensor. Due to the very small size and high current at low concentration of $\mathrm{DO}_{2}$, the geometrically modified microsensors demonstrate potential for clinical and biomedical applications.

\section{Operating Principle Of An Amperometric Three- ELECTRODE SENSOR}

The three function regions of a three-electrode Clark sensor primarily include the redox reaction under kinetic control (region 1), the redox reaction under diffusion limited conditions (region 2), and additional redox reactions (region 3) [9]. While there is an exponential relation between the applied voltage and the measured current in regions 1 and 3 , the sensing current is reported to be independent of the applied voltage in diffusion limited region (region 2). The developed $\mathrm{DO}_{2}$ sensors are characterized in region 2, since the measured current of the sensor is independent of the applied voltage and dependent of $\mathrm{DO}_{2}$ concentration in this region [9]. In a conventional Clark sensor, the sensing amperometric current is mathematically expressed in the diffusion limited region as (1).

$$
I=n F A D \frac{\partial C(x, t)}{\partial x}
$$

where $I$ is the amperometric current (A), $n, F, \mathrm{~A}, D$, and $C$ $(x, t)$ are the number of the reduced oxidised electrons, Faraday constant $(\mathrm{C} / \mathrm{mol})$, the area of electrode $\left(\mathrm{cm}^{2}\right)$, diffusion constant and the concentration of electroactive species at distance $\mathrm{x}$ and time $\mathrm{t}$, respectively.

The sensing amperometric current can be varied by varying the geometric area of the reference electrode. To show this, the operating principle of the conventional three-electrode sensor is discussed. Fig.1 shows the structure of a conventional three-electrode Clark sensor and the static couplings between the electrodes.

Considering $g_{t}$ as the total static capacitive coupling coefficient between the WE and other electrodes (RE and CE) in the sensor, $g_{t}$ is split into two static capacitive couplings: $g_{w r}$ (capacitive coupling coefficient between WE and RE) and $g_{w c}$ (capacitive coupling coefficient between WE and CE) [21].

$$
g_{t}=g_{w r}+g_{w c}
$$

However, the coupling coefficients, $g_{w r}$ and $g_{w c}$ depend on the geometric configuration of the electrodes. As schematically shown in Fig.1, a ratio of the amperometric current (conducted by electrochemical species from WE to $\mathrm{CE}$ ), is coupled to the RE which has low electric potential and corresponds to the capacitive coupling coefficient of $g_{c r}$. 
Taking the coupling coefficient of $g_{c r}$ into account, the capacitive coupling coefficients between WE-CE and WE-RE can be modified as follows:

$$
\begin{gathered}
g_{w c}^{\prime}=g_{w c}-g_{c r} \\
g_{w r}^{\prime}=g_{w r}+g_{c r}
\end{gathered}
$$

According to (3)-(4), the capacitive coupling coefficient $g_{c r}$ negatively contributes to the coupling coefficient between WE and $\mathrm{CE}$ and subsequently reduces the output amperometric current, $I$ between these two electrodes. In order to increase the output current, $I$, the capacitive coupling coefficient of $g_{c r}$ should be reduced $\left(\downarrow g_{c r} \rightarrow g_{w c}^{\prime} \uparrow\right)$ [21]. The capacitive coupling coefficient $g_{c r}$ is positively correlated to the surface area of RE in the structure $(a \times b$ in Fig.1). This coupling coefficient can be reduced by area reduction of the reference electrode. Moreover, the RE causes interference by creating resistance between the $\mathrm{WE}$ and $\mathrm{CE}$. Reducing the area of $\mathrm{RE}$ will reduce this resistance resulting in less interference for the output current, $I$.

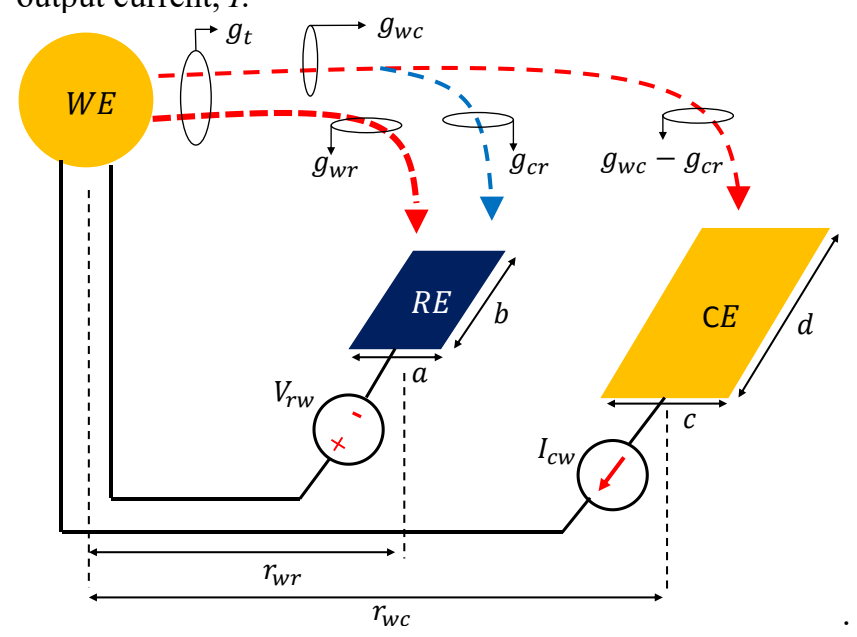

Fig. 1. Structure of a conventional three-electrode Clark sensor and the static couplings between the electrodes.

\section{Optimized Geometric Area Of Reference ELECTRODE FOR INTERFERENCE REDUCTION}

A conventional three electrode Clark microsensor as shown in Fig. 2(a) has a full crescent shaped reference electrode sandwiched between working (circle at the center) and counter (the exterior crescent) electrodes. To demonstrate the variations of the capacitive cross-coupling and resistance between electrode pairs (WE-RE pair and WE and CE pair), a concentric three-electrode Clark microsensor structure was simulated by COMSOL Multiphysics Modeling Software. The size of the reference electrode was reduced by taking off $1 / 4^{\text {th }}$ of the crescent (reducing the area by 1/4) at every pass. Therefore, the sensor structure was simulated for four different areas of the RE. Normalized to the conventional circular shape structure, the surface area of the reference electrode was reduced to the ratios of $3 / 4\left(3 / 4^{\text {th }}\right.$ crescent shape), $1 / 2$ (half crescent shape) and $1 / 4$ (quarter crescent shape) As shown in Fig. 2(b), (c) and (d). In the simulation, the capacitance and resistance variations between WE-RE and WE-CE pairs were calculated by applying a constant voltage of $1 \mathrm{~V}$ across the working and reference electrodes.

Fig. 3(a) compares the simulated capacitances between the WE and $\mathrm{RE}\left(C_{w r}\right)$ and $\mathrm{WE}$ and $\mathrm{CE}\left(C_{w c}\right)$ electrodes for different surface areas of the reference electrode in the structure. Simulation results indicates that as the surface area of the reference electrode decreases, the capacitance, $C_{w r}$ decreases while the capacitance $C_{w c}$ simultaneously increases. In a similar way, the resistance variations between WE and RE $\left(R_{w r}\right)$, and WE and CE $\left(R_{w c}\right)$ electrode pairs were simulated for different areas of RE (see Fig. 3(b)). The simulated results indicate that as the surface area of the RE decreases, the resistance $R_{w r}$ increases while the resistance $R_{w c}$ decreases. The simulated results in figs. 2 and 3 indicate that a RE with reduced surface area has less capacitive coupling coefficient $g_{c r}$ and contributes to a high output current, I. Finally, the output current $I$ (the current between the WE and CE) was simulated for different reference electrode surface area. Fig. 3(c) shows that the output current is maximum when the surface area of RE chosen to be the half of the conventional re surface area.

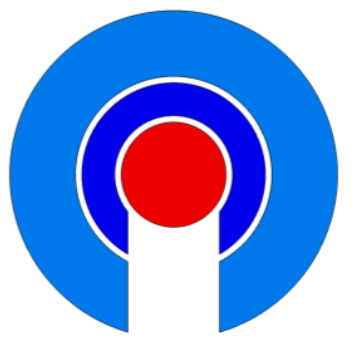

(a)

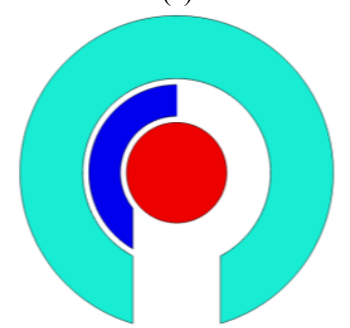

(c)

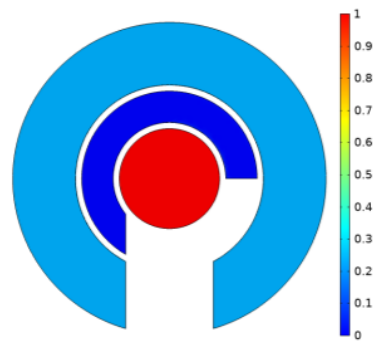

(b)

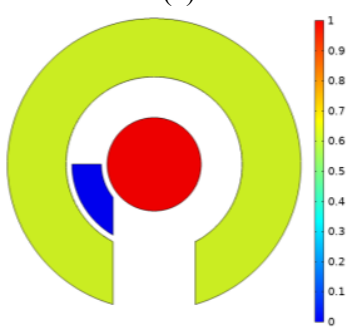

(d)
Fig. 2. Topology of the three-electrode Clark sensor for different reference electrode area with normalized area of (a) 1 (b) $3 / 4$ (c) $1 / 2$ (d) $1 / 4$. 


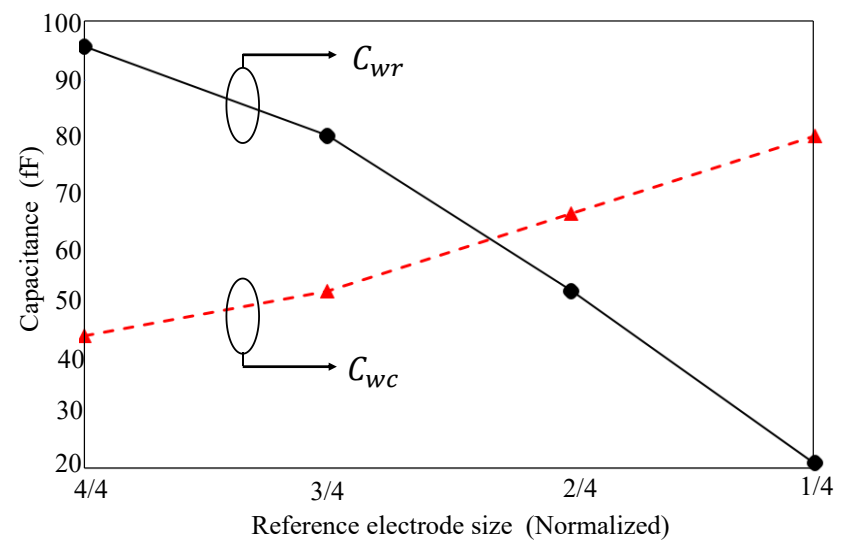

(a)

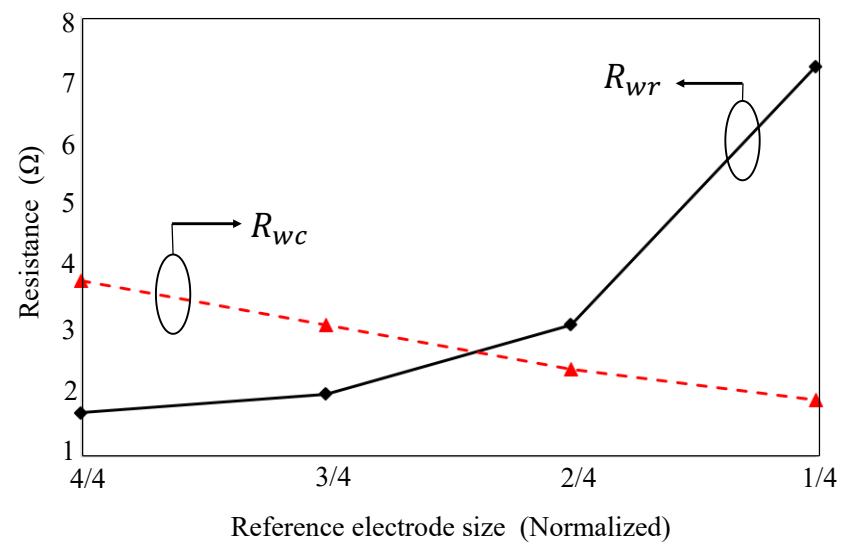

(b)

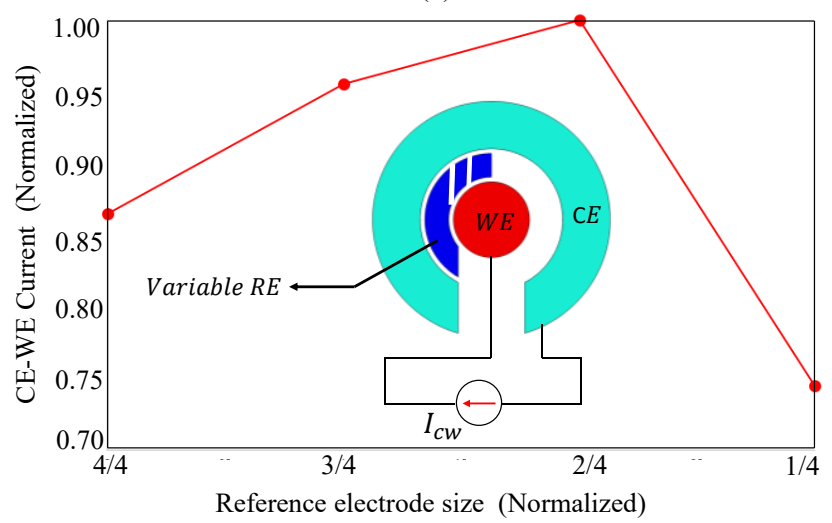

(c)

Fig. 3. Simulated (a) capacitance variations between WE-RE $\left(C_{w r}\right)$ and WECE $\left(C_{w c}\right)$, (b) resistance variations between WE-RE $\left(R_{w r}\right)$ and WE-CE $\left(R_{w c}\right)$ and (c) output current variations between WE-CE $(I)$ for different surface area of reference electrode.

\section{TYPEI DO 2 MICROSENSOR}

\section{A. Microfabrication}

For the miniaturization requirement of the implantable biomedical applications, the $\mathrm{DO}_{2}$ microsensor was fabricated on a silicon wafer. Based on the design procedure in Section III, a concentric three-electrode Clark microsensor with optimized half crescent-shaped reference electrode (type I $\mathrm{DO}_{2}$ microsnesor) was designed and fabricated. To facilitate comparison, a conventional Clark $\mathrm{DO}_{2}$ microsensor was also designed and fabricated. The conventional Clark microsensor and Type I microsensor have same dimension everywhere except for the reference electrode except reference electrode shape. The reference electrode was a full crescent and half crescent for the conventional Clark $\mathrm{DO}_{2}$ microsensor and the type $\mathrm{I} \mathrm{DO}_{2}$ microsensor, respectively. Figs. $4 \mathrm{a}$ and $4 \mathrm{~b}$ show the layout of the conventional Clark and type I microsensors, respectively and $4 \mathrm{c}$ shows the cross section of the microsensors on a (100)-oriented silicon wafer.

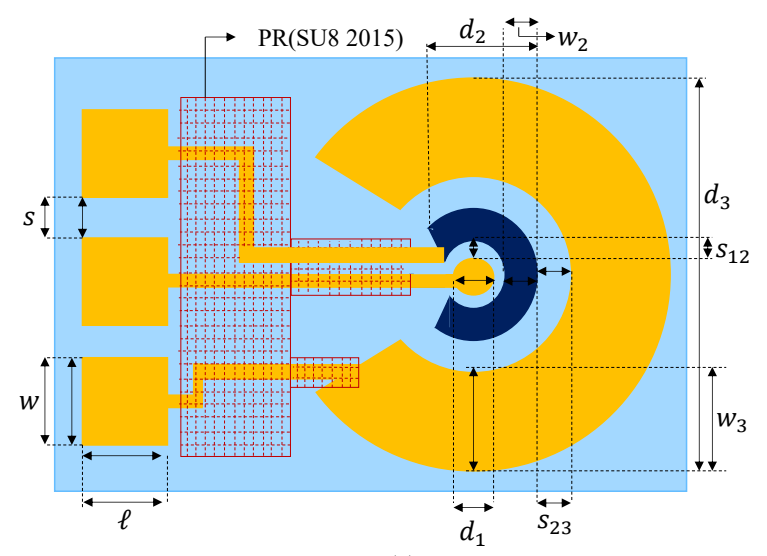

(a)

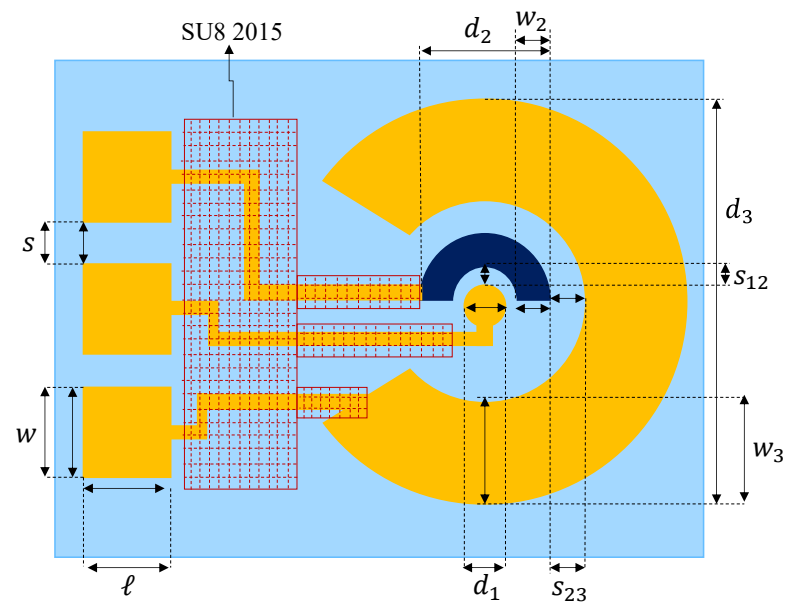

(b)

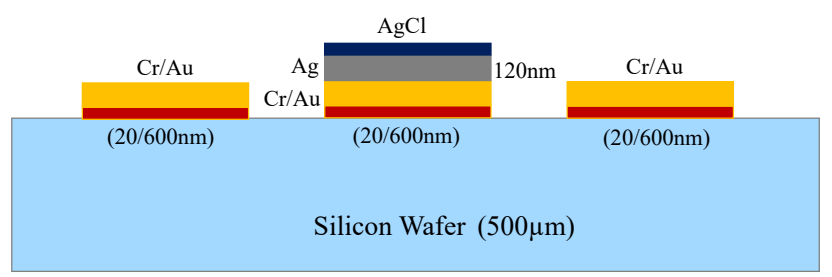

(c)

Fig. 4. Layout of (a) the conventional Clark $\mathrm{DO}_{2}$ microsensor and (b) the type I $\mathrm{DO}_{2}$ microsensor. (c) Cross section of both sensors with $d_{1}=100, d_{2}=300$, $d_{3}=900, \ell=280, s=50, w=270, w_{2}=80, w_{3}=280$ and $s_{12}=s_{23}=20$ (all dimensions in $\mu \mathrm{m})$.

Both $\mathrm{DO}_{2}$ sensors were fabricated following the same processes. A thin layer of 20-nm-thick chromium $(\mathrm{Cr})$ was initially deposited with electron-beam-sputtering at $300^{\circ} \mathrm{C}$ and $10^{-7}$ Torr. It was followed by electron-beam-sputtering of 600 nm-thick gold (Au) layer at $300^{\circ} \mathrm{C}$ and $10^{-7}$ Torr. The Cr layer acts an adhesion layer between the base silicon substrate and electron-beam-sputtered $\mathrm{Au}$ layer. The $\mathrm{Au}$ and $\mathrm{Cr}$ layers were 
lithographically patterned by wet chemical etching process. Then a thin layer of 200-nm-thick LOR 2A photoresist followed by a $1.5-\mu \mathrm{m}$-thick $\mathrm{S} 1813$ photoresist were spin coated and pattered on the structure for the bi-layer lift-off process of silver layer. Next, a 120-nm-thick silver layer was deposited on the bi-layer photoresists using chemical vapor deposition at $220^{\circ} \mathrm{C}$ and $5 \times 10^{-6}$ Torr. The $\mathrm{Ag}$ layer was then lithographically patterned by lift-off process. The etching of materials had to be done properly so that there is was no metallic contact between the electrodes and the connection pads of the electrodes. Then SU-8 2015 photoresist was spincoated and patterned following soft baking, exposure, post exposure baking and development. This resulted in a $300 \mu \mathrm{m}$ SU-8 2015 layer covering the connections between the sensing area and connection pads. The patterning of SU-8 2015 needed to be done with care so that electrodes and connection pads were not covered with SU-8 2015. Finally, the deposited silver layer $(\mathrm{Ag})$ is converted to $\mathrm{Ag} / \mathrm{AgCl}$ by traditional electrochemical deposition technique. The $\mathrm{AgCl}$ layer was formed on the $\mathrm{Ag}$ layer by applying a $5 \mathrm{uA}$ current for $15 \mathrm{sec}$ in a $3 \mathrm{M}$ potassium chloride $(\mathrm{KCl})$ solution against a $\mathrm{Pt}$ counter electrode (also serving as reference in this case).

Fig. 5 shows the digital photograph of the fabricated conventional and type $\mathrm{I} \mathrm{DO}_{2}$ microsensors on a silicon wafer. Including the connection pads, each $\mu$-sensor occupies a surface area of $2 \mathrm{X} 1 \mathrm{~mm}^{2}$. Once the sensors were made, the sensors were mounted on a designed PCB by connecting 150 $\mu \mathrm{m}$ diameter copper wires between the connection pads of rge sensor and the PCB (shown in Fig. 6). The thin copper wires were connected to the connection pads with conductive epoxy. Since the distance between connection pads on the microsensors was $50 \mu \mathrm{m}$, care had to be taken so that the conductive epoxy does not short the microsensor connection pads. After successful electrical connections were made, the connection areas and the copper wires were covered with insulating materials so that only the sensing area of the sensors was exposed to test liquids.

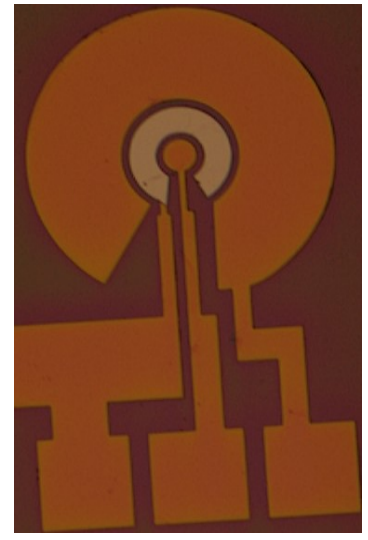

(a)

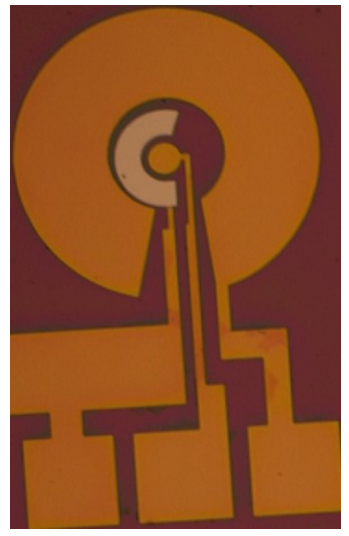

(b)
Fig. 5. Digital photographs of the fabricated (a) conventional and (b) type I $\mathrm{DO}_{2}$ microsensors on a silicon wafer.

\section{B. Experimental Results}

Electrochemical experiments were performed in a threeneck glass reactor vessel as shown in Fig. 6. The glass reactor vessel was filled with $0.5 \mathrm{M}$ phosphate buffer (PBS, $\mathrm{pH} 7$ ) solution. The microsensor (either the type $\mathrm{I}$ or the conventional Clark) was placed in the solution through neck 2 . The solution was saturated with different oxygen/nitrogen gas ratios at room temperature with a tube placed through neck 1. For reference, $\mathrm{DO}_{2}$ in the bulk solution was monitored with a commercial oxygen meter Instech, SYS203 placed through neck 3. Experiments were performed with a computer driven low-current potentiostat (Gamry Instruments, FAS1) in a three-electrode configuration at room temperature. Fig. 7(a) shows the cyclic voltammogram (CV) of the conventional Clark and type I microsensors for a $96 \%$ of $\mathrm{DO}_{2}$ solution. The bias potential between the working and reference electrodes was swept at a rate of $-5 \mathrm{mV} / \mathrm{s}$.

To measure the chronoamperometric response, the cathodic potential for oxygen reduction $-0.75 \mathrm{~V}$ versus $\mathrm{Ag} / \mathrm{AgCl}$ was chosen from the plateau region. Typical chronoamperometric responses were first observed at the onset of potential pulses of $-0.75 \mathrm{~V}$ to the WE. Afterward, the current reached a steadystate value known as oxygen limiting current. Fig. 7(b) provides the experimental chronoamperometry response of the conventional Clark and type $\mathrm{I} \mathrm{DO}_{2}$ microsensors for $96 \% \mathrm{DO}_{2}$ solutions. The amperometric currents between WE and CE, $I_{c w}$, were measured to be around $0.27 \mu \mathrm{A}$ and $0.87 \mu \mathrm{A}$ for the conventional Clark and type I microsensors, respectively. Fig. 8 shows the chronoamperometric responses of the conventional Clark and type I microsensors for three different concentrations, $2 \%, 23 \%$ and $96 \%$ of $\mathrm{DO}_{2}$. It can be seen that the type $\mathrm{I} \mathrm{DO}_{2}$ microsensor has higher current output than conventional Clark $\mathrm{DO}_{2}$ microsensor.

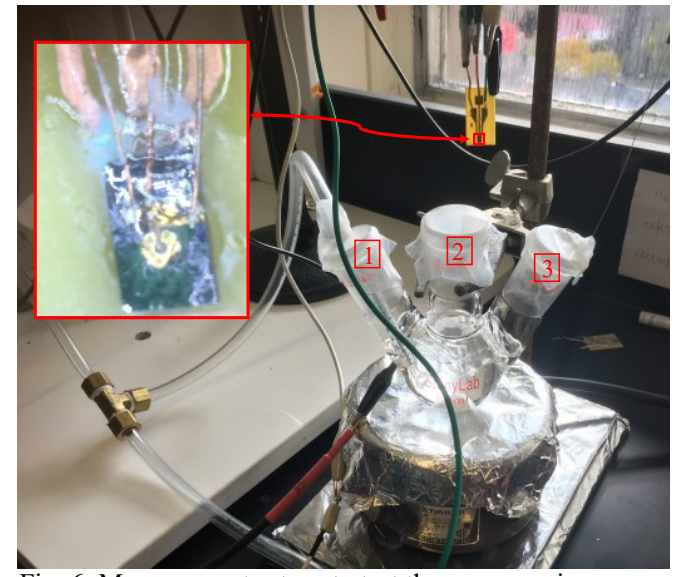

Fig. 6. Measurement set-up to test the oxygen microsensors. 


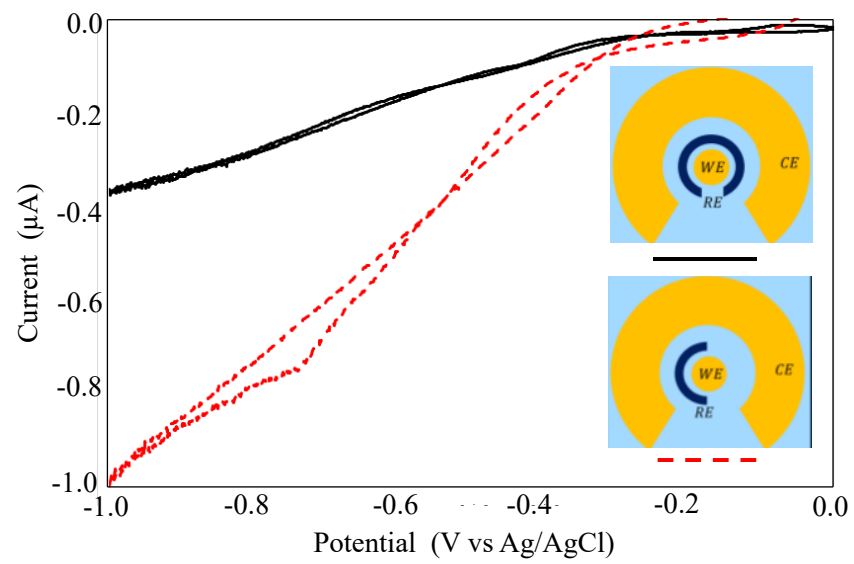

(a)

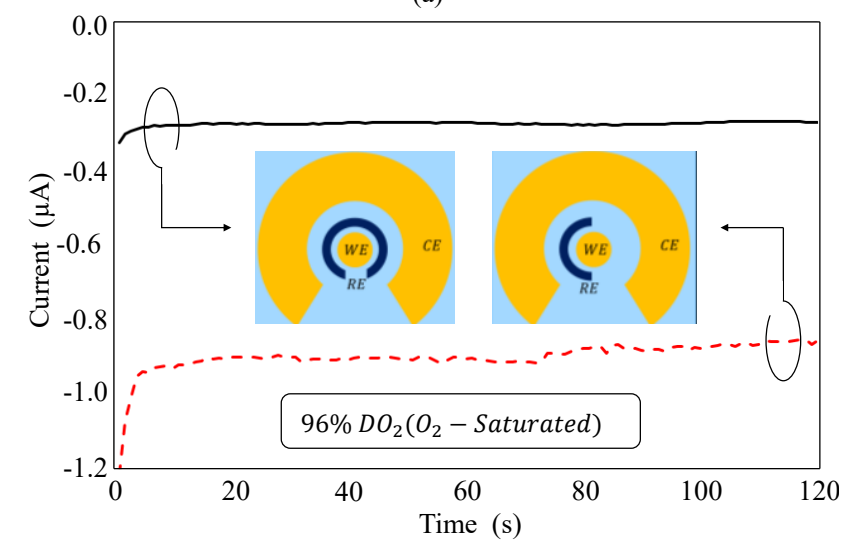

(b)

Fig. 7. (a) Cyclic voltammogram (CV) and (b) time responses (chronoamperometry, $I_{c w}$ ) of the conventional Clark and type I microsensors for $96 \% \mathrm{DO}_{2}$.

Fig. 9 shows the measured current across working and counter electrodes versus the dissolved oxygen concentration measured with the commercial oxygen probe for both microsensors. The current was measured four times for each concentration of $\mathrm{DO}_{2}$ (removing the oxygen gas and saturating the solution with nitrogen gas in between those four chronoamperometry measurements), totaling upto 12 chronoamperometry current measurements. Each data point in the plot is the average of chronoamperometry current after the current reaches to a stable value (after 8 seconds). Linear fits to the data show that the type I sensor has a sensitivity of $0.0094 \mu \mathrm{A} / \% \mathrm{DO}_{2}$ and the conventional Clark sensor has a sensitivity of $0.0027 \mu \mathrm{A} / \% \mathrm{DO}_{2}$. The results show an enhanced sensitivity coefficient of 3.48:1 for the type I $\mathrm{DO}_{2}$ microsensor compared to the conventional Clark one. Additionally, it can be concluded that the type I sensor demonstrates an accuracy of $1.98 \% \mathrm{DO}_{2}$, a precision of $4 \% \mathrm{DO}_{2}$ and a resolution of $0.004 \% \mathrm{DO}_{2}$. For precision measurement, spread of data for each concentration was considered and the maximum spread was taken as precision. It should be noted that errors were introduced in measurement and instrumentation process. Electrolyte resistance and resistance of other components such as electrical connection to the working electrode in the electrochemical circuits can have a significant effect on measurements [22]. Cautions were taken to make these resistances as small as possible for measurement accuracy. In the tests, bubbles were created when gases were passed in the buffer solution. Bubbles cover parts of the working electrode surface, increasing over-potential on the working electrode's uncovered parts and taking up an appreciable volume fraction of electrolyte. This changes the effective electrolyte conductivity [22]. Thus the bubbles were a source of measurement error. Another important source of error in measurement came from the control amplifier inside the potentiostat. DC signals applied by the control amplifier are always superimposed by a very small AC signal consisting of ripple and noise. The ripple and noise distort the applied DC signal to the electrodes and produce measurement error. Finally, our measurements had DC offset error. This error is the drift of DC performance of the potentiostat over time and with temperature change. For our tests, potentiostat was used for long time to perform multiple measurements with different types of sensor. Additionally, the tests were performed at room temperature which varied around $2^{\circ} \mathrm{C}$ during the entire testing period.

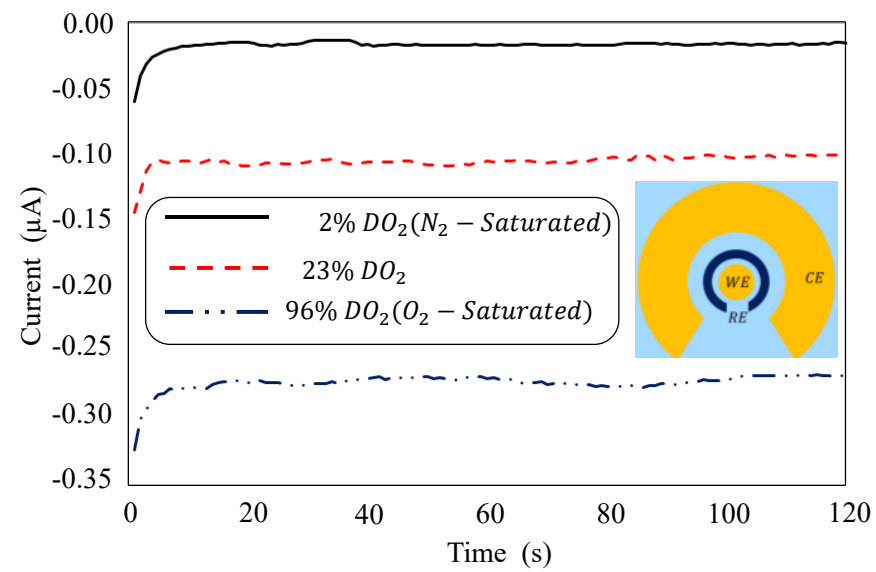

(a)

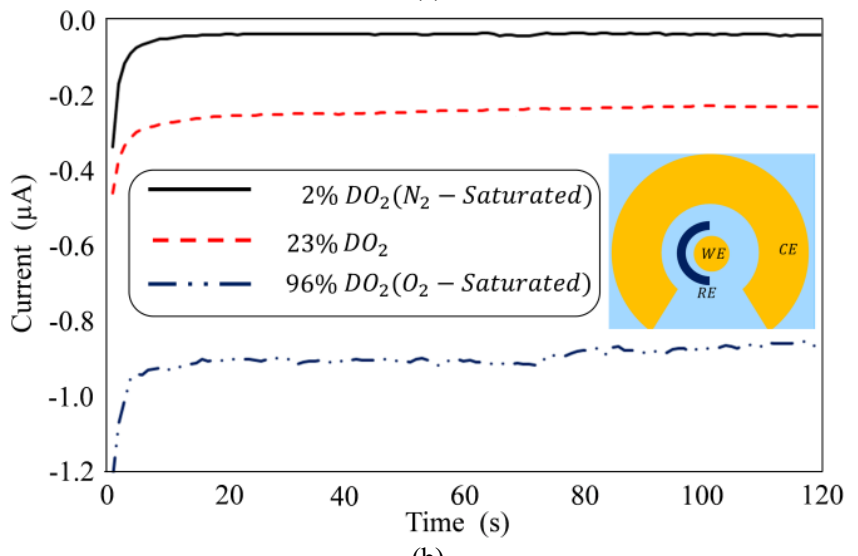

(b)

Fig. 8. Chronoamperometric responses of (a) the conventional Clark and (b) type I microsensors for three different concentrations, $2 \%, 23 \%$ and $96 \% \mathrm{DO}_{2}$. 


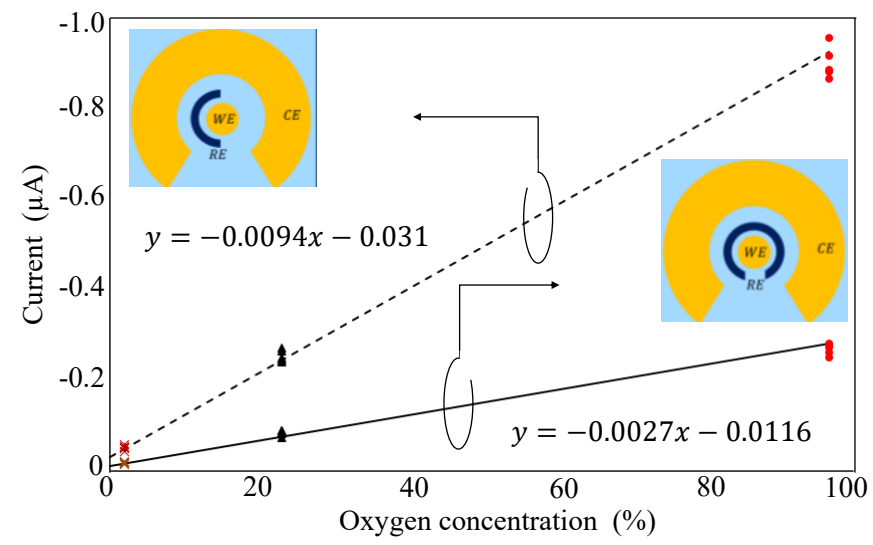

Fig. 9. Relationship between calibrated $\mathrm{DO}_{2}$-concentrations and the measured current across working and counter electrodes.

\section{TYPE I I DO 2 MICROSENSOR}

\section{A. Operating Principle}

Inspecting the geometric configuration of the threeelectrode conventional Clark sensor in Fig. 1 it is obvious that a ratio of the capacitive coupling between $\mathrm{WE}$ and the exterior $\mathrm{CE}, g_{w c}$, is coupled to the reference electrode and is denoted by capacitive coupling coefficient, $g_{c r} \cdot g_{c r}$ positively contributes to the capacitive coupling between the WE and RE while it negatively contributes to the capacitive coupling between the WE and CE (see equations (3)-(4)). For type I $\mathrm{DO}_{2}$ microsensor, the magnitude of this coupling coefficient was reduced to some extent by reducing the surface area of the reference electrode. For type II microsensor the geometry of the sensor is modified to invert the contribution of coupling coefficient, $g_{c r}$ in favor of output current of the sensor.

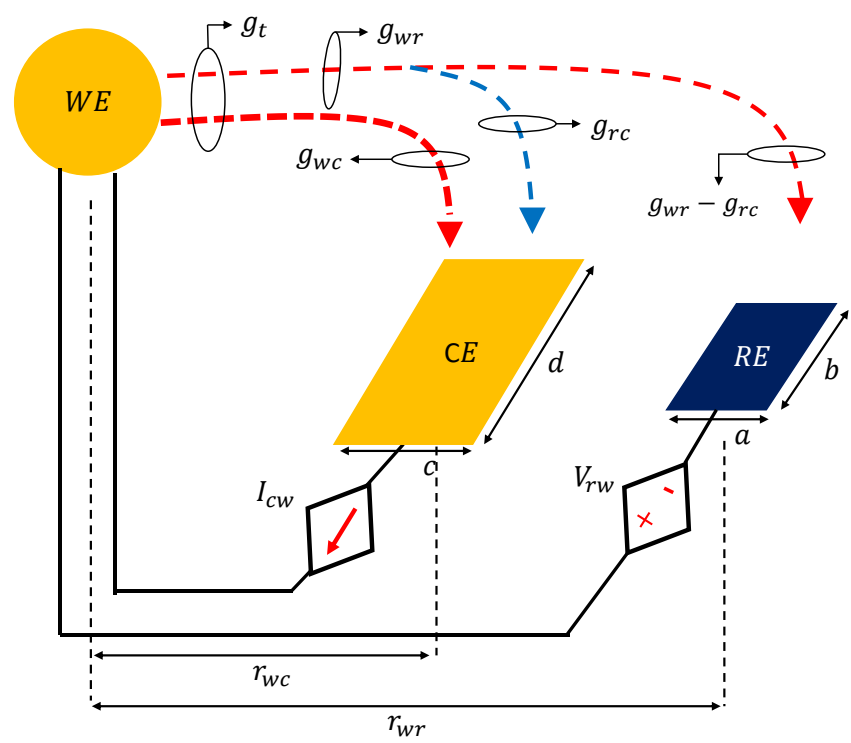

Fig. 10. Static couplings between the electrodes in a geometrically modified three-electrode Clark sensor.

The sensing amperometric current can be significantly enhanced by sandwiching $\mathrm{CE}$ in between $\mathrm{WE}$ and $\mathrm{RE}$ as shown in Fig. 10. In this configuration a ratio of the amperometric current transported by electrochemical species from WE to RE is coupled to the CE (see Fig. 10). This ratio of the current corresponds to the capacitive coupling coefficient $g_{r c}$. Considering the capacitive coupling coefficient $g_{r c}$ in Fig. 10, the capacitive coupling coefficient between WE-CE and WE-RE is modified as follows:

$$
\begin{gathered}
g_{w c}^{\prime \prime}=g_{w c}+g_{r c} \\
g_{w r}^{\prime \prime}=g_{w r}-g_{r c}
\end{gathered}
$$

Comparing the two sets of equations (3)-(4) and (5)-(6), the capacitive coupling coefficient $g_{c r}$ negatively contributes to output amperometric current, $I$ of the conventional structure of the sensor, while the capacitive coupling coefficient $g_{r c}$ positively contributes to this $I$ in the topologically modified three-electrode Clark sensor. Moreover, in the modified structure, the distance between $\mathrm{WE}$ and $\mathrm{CE}, r_{w c}$, is reduced which positively contributes to capacitive coupling between these two electrodes. Therefore, to enhance the sensing amperometric current, $I$ in type II microsensor $\mathrm{CE}$ is sandwiched between WE and RE.

\section{B. Microfabrication}

Based on our findings in section V.A, a concentric three electrode Clark micro sensor with the CE sandwiched between the WE and RE was designed and fabricated on a silicon wafer. Fig. 11 shows the schematic diagram of the microsensor on a (100)-oriented silicon. The sensor was fabricated using the same microfabrication steps as described in section IV.A for the type I and conventional Clark $\mathrm{DO}_{2}$ microsensor. The thickness of each layer was same as the thickness of the layers for the type I and conventional Clark $\mathrm{DO}_{2}$ microsensor. Including the connection pad region, the dimension sensor area is $2 \mathrm{X} 1 \mathrm{~mm}^{2}$.

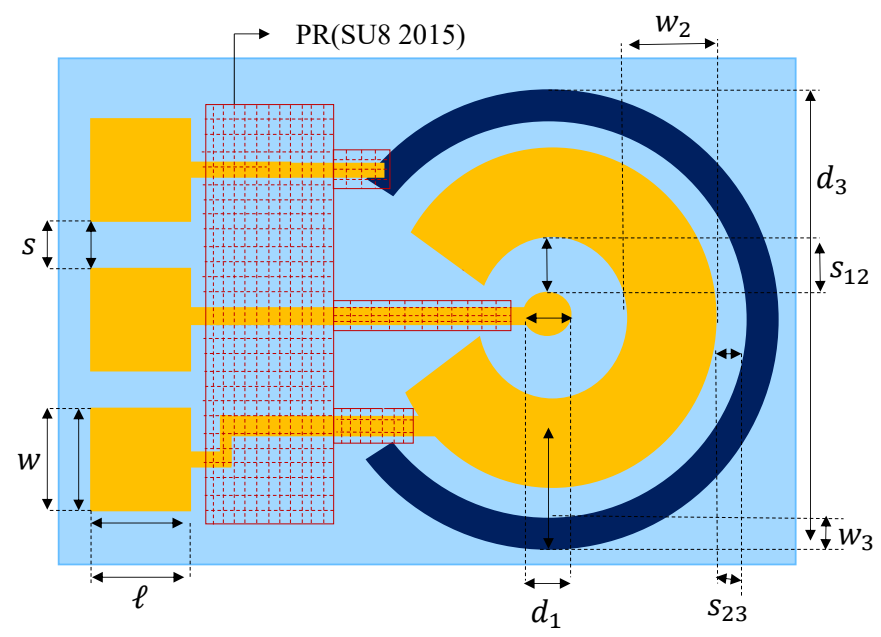

Fig.11. Layout of the type II $\mathrm{DO}_{2}$ microsensor with the optimized $d_{1}=100$, $d_{2}=300, d_{3}=900, \ell=280, s=50, w=270, w_{2}=280, w_{3}=80$ and $s_{12}=s_{23}=20$ (all dimensions in $\mu \mathrm{m})$. 


\section{Experimental Results}

To verify the performance of the type II sensor in Fig.11, the same experimental set-up used in section V.B was used. Fig.12(a) shows the linear cyclic voltammogram (CV) of the type II microsensor for two specific dissolved oxygen solutions (N2- and O2-Saturated). Fig. 12(b) compares the measured CV curve of the type II microsensor with the CV curves of type I and conventional Clark sensor previously discussed in section $\mathrm{V}$ for $96 \% \mathrm{DO}$. It can be seen that a significant enhancement in the sensing amperometric current was achieved with type II microsensor, e.g., the measured sensing current was around $-2.35 \mu \mathrm{A}$ for the type II microsensor versus $-0.87 \mu \mathrm{A}$ and $-0.27 \mu \mathrm{A}$ for type I and conventional clark sensor, respectively for $96 \%$ DO2 at -0.75 V. Fig.13 shows the chronoamperometric responses of the type II microsensor with the measured currents of $-0.13 \mu \mathrm{A}$, $0.64 \mu \mathrm{A}$ and $-2.35 \mu \mathrm{A}$ corresponding to three different concentrations, $2 \%, 23 \%$ and $96 \% \mathrm{DO} 2$, respectively. To measure the chronoamperometric response, the cathodic potential for oxygen reduction ( $-0.8 \mathrm{~V}$ versus $\mathrm{Ag} / \mathrm{AgCl}$ ) was chosen from the plateau region.

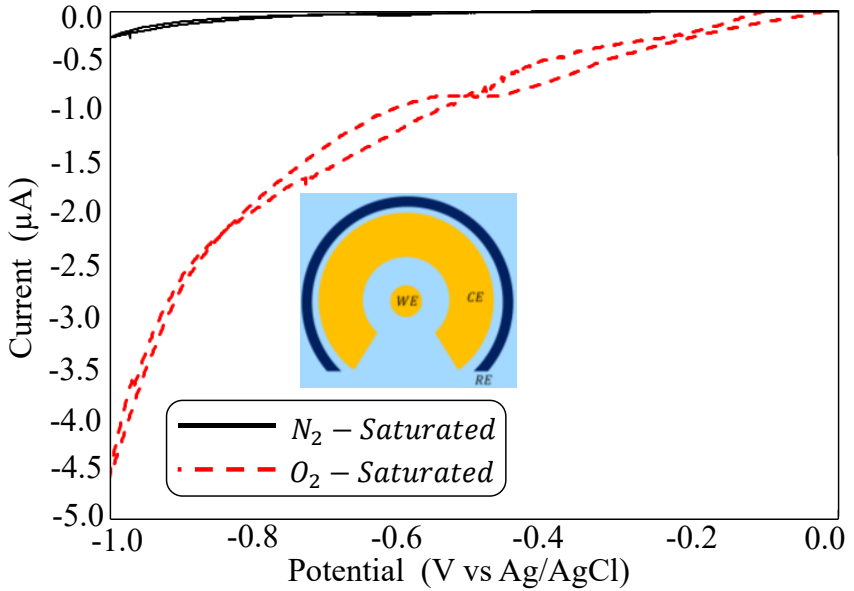

(a)

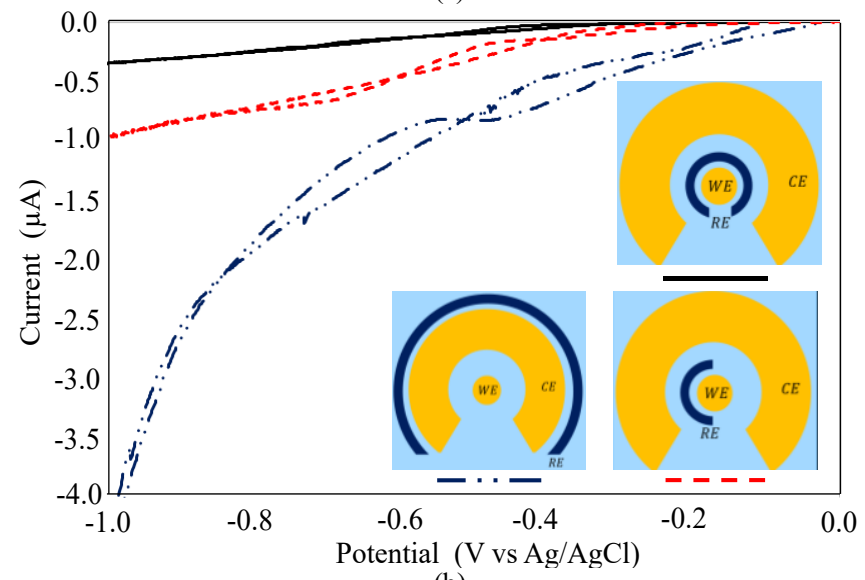

(b)

Fig. 12. (a) Linear cyclic voltammogram (CV) of the type II microsensor for $\mathrm{N}_{2}$ - and $\mathrm{O}_{2}$ - saturated condition. (b) comparison between $\mathrm{CV}$ responses of three microsensor sensors for $96 \% \mathrm{DO}_{2}$.

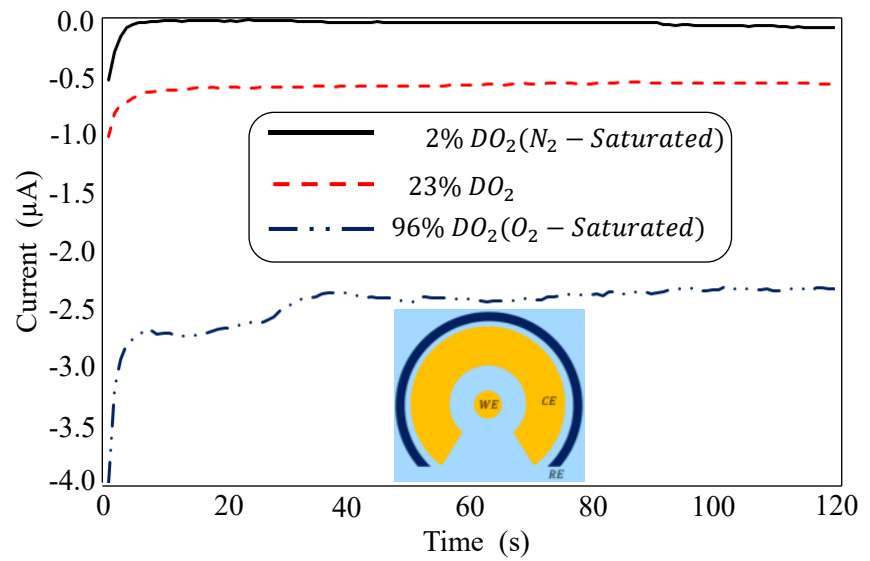

Fig. 13. Chronoamperometric responses of the type II microsensor for three different concentrations, $2 \%, 23 \%$ and $96 \% \mathrm{DO}_{2}$.

Fig. 14 shows the measured current across working and counter electrodes versus the $\mathrm{DO}_{2}$ concentration measured with a commercial oxygen probe for type II microsensor. The current was measured four times for each concentration of $\mathrm{DO}_{2}$ (removing the oxygen gas and saturating the solution with nitrogen gas in between those four chronoamperometry measurements). Each data point in the plot is the average of chronoamperometry current after the sensor reaches to a stable value (after 40 seconds). Linear fits to the data show that the type II microsensor has a sensitivity of $-0.024 \mu \mathrm{A} / \% \mathrm{DO}_{2}$. The results show an enhanced sensitivity coefficient of 8.9:1 for the type II microsensor compared to the conventional Clark microsensor. The accuracy, precision and resolution of the type II microsensor are computed to be $2.1 \% \mathrm{DO}_{2}, 6 \% \mathrm{DO}_{2}$ and $0.006 \% \mathrm{DO}_{2}$, respectively. For precision measurement, spread of data for each concentration was considered and the maximum spread was taken as precision. The sources of errors in measurement and instrumentation process are same as discussed in section IV. B.

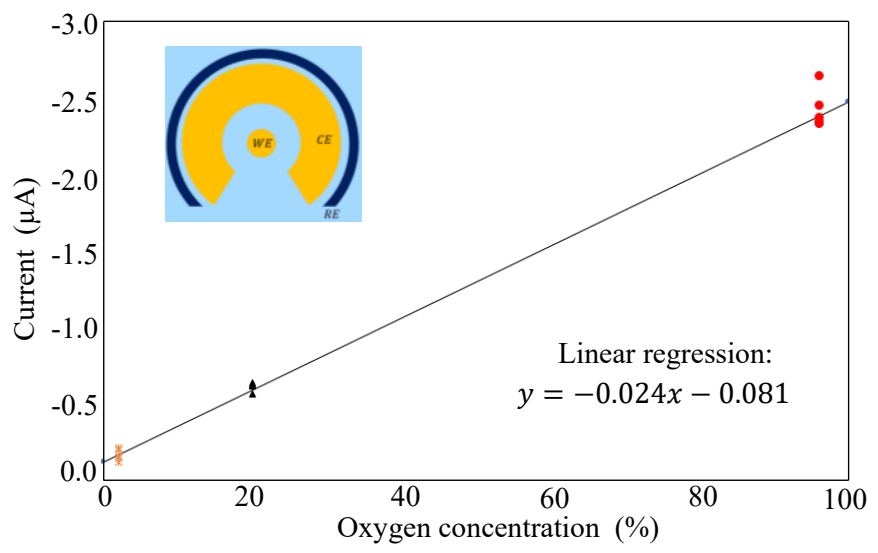

Fig. 14. Relationship between calibrated $\mathrm{DO}_{2}$-concentrations and the measured current across working and counter electrodes.

Table I compares the developed geometrically modified $\mu$ sensor with the reported $\mathrm{DO}_{2}$ microsensors in literature in terms of size, technology, sensitivity coefficient and the measured amperometric current at $\mathrm{O}_{2}$-saturate concentration. The developed type II microsensor is reported to have higher measured current than the ones reported in the literature 
except the one reported in in [19]. However, the sensor reported in [19] achieves higher current at the expense of a large size which is not suitable for many clinical and biomedical application.

TABLE I

COMPARISON BETWEEN THE GEOMETRICALLY MODIFIED DO2 MICROSENSORS AND MICROSENSORS IN LITERATURE

\begin{tabular}{ccccc}
\hline \hline Sensor & Size $\left(\mathrm{mm}^{2}\right)$ & Tech. & $\begin{array}{c}\text { Sensitivity } \\
\text { Coefficient }\end{array}$ & $\begin{array}{c}\text { Current } \\
(\mu \mathrm{A}) \mathrm{O}_{2^{-}} \\
\text {Saturated }\end{array}$ \\
\hline$[2]$ & $3.5 \times 6.5$ & MEMS & Not Given & 0.31 \\
{$[11]$} & $0.2 \times 0.5$ & MEMS & Not Given & 0.27 \\
{$[23]$} & $12 \times 20$ & MEMS & Not Given & 0.14 \\
{$[18]$} & $4.0 \times 4.0$ & MEMS & $0.0014 \mathrm{nA} / \mathrm{ppm}$ & 0.04 \\
{$[19]$} & $20 \times 40$ & PCB & Not Given & 4.10 \\
{$[20]$} & $3.0 \times 3.0$ & LTCC & $0.005 \mu \mathrm{A} / \% \mathrm{DO}_{2}$ & 0.41 \\
{$[5]$} & $2.0 \times 6.0$ & MEMS & $0.011 \mu \mathrm{A} / \% \mathrm{DO}_{2}$ & 1.68 \\
Type I & $1.0 \times 2.0$ & MEMS & $0.009 \mu \mathrm{A} / \% \mathrm{DO}_{2}$ & 0.87 \\
Type II & $1.0 \times 2.0$ & MEMS & $0.024 \mu \mathrm{A} / \% \mathrm{DO}_{2}$ & 2.35 \\
\hline
\end{tabular}

Both type I and type II sensors were fabricated on two different wafers using the same microfabrication processes at two different times. Five sensors of both types from each wafer were tested. Table II shows the measured amperometric current of both type sensors fabricated on two different wafers for $96 \% \mathrm{DO}_{2}\left(\mathrm{O}_{2}-\right.$ saturated $)$. It can be seen that the amperometric currents of same type sensors fabricated on two different wafers have very small deviation between them. This indicates that reproducible sensors can be fabricated if the same microfabrication processes are followed.

TABLE II

AMPEROMETRIC CURRENT OF BOTH TYPE SENSORS FABRICATED ON TWO DIFFERENT WAFERS

\begin{tabular}{ccc}
\hline \hline Wafer & Type & $\begin{array}{c}\text { Current } \\
(\mu \mathrm{A}) \mathrm{O}_{2^{-}} \\
\text {Saturated }\end{array}$ \\
\hline Wafer 1 & Type I & $0.87 \pm 0.02$ \\
Wafer 2 & Type I & $0.88 \pm 0.05$ \\
Wafer 1 & Type II & $2.35 \pm 0.05$ \\
Wafer 2 & Type II & $2.32 \pm 0.06$
\end{tabular}

\section{Stability Of The MicRosensors}

Developed Type I and Type II microsensors were tested to analyze the long-term stability performance. For the test, the same experimental set-up used in section V.B was used. The sensors were tested in a $0.5 \mathrm{M}$ phosphate buffer (PBS, pH 7) solution saturated with oxygen. The cyclic voltammogram (CV) performed periodically every hour at room temperature for a total of approximately 64 hours. Fig. 15 shows the output currents of type I and type II microsensors measured at $-0.75 \mathrm{~V}$ versus $\mathrm{Ag} / \mathrm{AgCl}$ and $-0.8 \mathrm{~V}$ versus $\mathrm{Ag} / \mathrm{AgCl}$ for type I and type II microsensors, respectively. As shown in Fig. 15, the output current remained stable over the duration of 53 hours after which an increase in current drift was observed. The measured currents lied with in $-0.87 \pm 0.04 \mu \mathrm{A}$ and -2.35 $\pm 0.07 \mu \mathrm{A}$. It is speculated that the increased drift after 53 hours is due to the degradation of $\mathrm{AgCl}$ layer on the RE. The microsensors can support testing for 2 days if one test is performed every hour. This lifetime is enough for some biomedical and clinical applications such as measuring brain tissue oxygenation of TBI patients. For traumatic brain injury (TBI) patients, monitoring brain tissue oxygenation level for first 48 hours is of great importance.

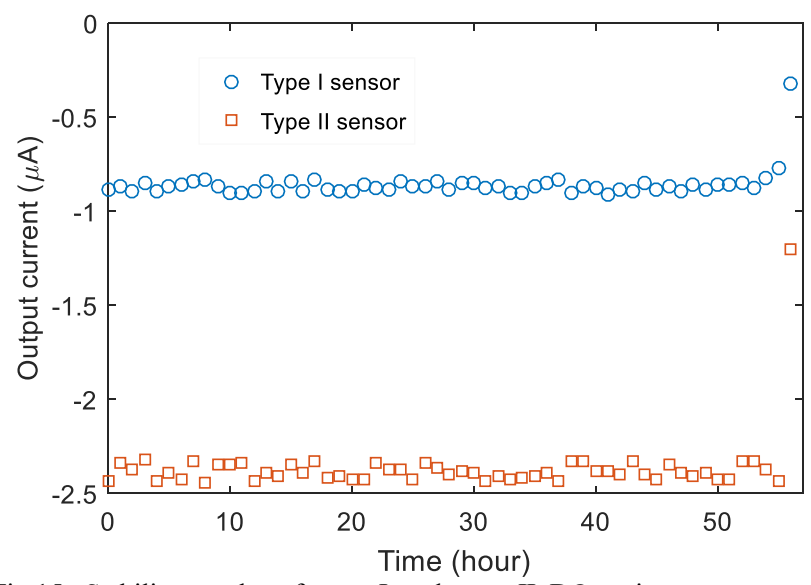

Fig.15. Stability results of type I and type II $\mathrm{DO}_{2}$ microsensors at room temperature for saturated with oxygen solution.

Studies have been done to improve the stability of reference electrode and increase lifetime of sensors. [24] has improved the stability of the reference electrode by improving stability of the $\mathrm{AgCl}$ layer on $\mathrm{Ag}$. They have applied a solid $\mathrm{KCl}$ layer on the $\mathrm{AgCl}$ layer and a protective nafion layer on the $\mathrm{KCl}$ layer. The protective nafion layer prevents the leakage of chloride ions into the solutions. [5] has improved the stability of reference electrode by choosing gold as reference electrode material. One of these approaches can be taken in future to increase the lifetime of the developed microsensors. Additionally, it should be noted that the results of the tests reported here did not take cross-sensitivity into account. The cross sensitivity of the sensor can be reduced by placing an electrolyte in contact with the electrodes and then encapsulating the electrolyte and electrodes with an oxygen gas permeable membrane [5].

\section{DISCUSSION AND CONCLUSION}

Three-electrode Clark $\mathrm{DO}_{2}$ microsensors are geometrically modified to enhance the sensing amperometric current and increase the sensitivity of measurements. Two types of microsensors have been designed and characterized. For type I sensor, the area of RE is optimized to increase the sensing current between WE-CE. For type II sensor the sensing current between WE-CE is significantly enhanced by sandwiching the CE between WE and RE. Both sensors have been fabricated using simple processes of microfabrication technology. The simple processes will result in low cost of the sensors. For $96 \% \mathrm{DO}_{2}$, type I and type II microsensors demonstrate a sensing current of $-0.87 \mu \mathrm{A}$ and $-2.35 \mu \mathrm{A}$ as compared to a sensing current of $-0.27 \mu \mathrm{A}$ for the conventional Clark microsensor of same size. Type I and type II have an enhanced sensitivity coefficient of 8.9:1 and 3.48:1, respectively compared to the conventional Clark microsensor of same size. With more than 48 hours lifetime and miniature size, the sensors have potentials for clinical and biomedical applications. 
Considering the cost associated with fabrication and packaging at the University, each type I or type II sensor costs around $35 \$$ when ready for testing. This is lower than the cost of bulky electrochemical $\mathrm{DO}_{2}$ probes in market (cost around 300-400\$). There are commercial optical DO2 sensors which are small (around $3 \mathrm{~mm}$ diameter) and low cost (around 33\$) [25]. They need to be attached to inner surface of vessels. However, these optical sensors can be used only for transparent vessels. Our developed sensors can be monolithically integrated with driving and reader CMOS electronics by post-processing route (fabricating the microsensors directly on top of CMOS) [26, 27]. There are two critical parameters to be addressed for post-CMOS integration of MEMS devices. First, the microfabrication process temperature budget must be lower $425^{\circ} \mathrm{C}$ in order to retain the performance and reliability of the CMOS electronics. Second, MEMS devices with gaps between structures need the sacrificial material and release etchant to be carefully chosen so that the metallization stack of the underlying electronics is saved from chemical [27]. The maximum processing temperature of our complete microsensors was kept below $300^{\circ} \mathrm{C}$ and the microsensor does not have any gaps between structures enabling above-CMOS integration in near future.

\section{REFERENCES}

[1] N. Akmal and J. Lauer, "Electrochemical oxygen sensors: Principles and applications," Polym. Sensors, vol. 690, pp. 149-160, Jan. 1998.

[2] D. Ivnitski, et.al, "Application of electrochemical biosensors for detection of food pathogenic bacteria," Electroanalysis, vol.12, no. 5, pp.317-325, Mar. 2000.

[3] E.J. Mahoney, "Optofluidic dissolved oxygen sensing with sensitivity enhancement through multiple reflections," IEEE Sensors J., vol. 19 , no. 22 , pp. $10452-10460$, Nov. 2019.

[4] A. A. Figaji, E. Zwane, C. Thompson et al., "Brain tissue oxygen tension monitoring in pediatric severe traumatic brain injury," Childs Nerv Syst, vol. 25, pp. 1325, Feb. 2009.

[5] D. She and M. G. Allen, "A micromachined freestanding electrochemical sensor for measuring dissolved oxygen," $J$. Microelectromech. Sys., vol.28, no.3, pp.521-531, Jun. 2019.

[6] M. Lambrechts and W. Sansen, Biosensors: Microelectrochemical Devices. Geneva, Switzerland: IOP, pp. 206-208, 1992.

[7] M. Wittkampf, et. al.,"Silicon thin film sensor for measurement of dissolved oxygen," Sens. Actuators B, Chem., vol. 43, nos. 1-3, pp. 4044, 1997.

[8] S-C. Liao, Z. Xu, J.A. Izatt, and J.R. Alcala, "Real-time frequency domain temperature and oxygen sensor with a single optical fiber," IEEE Trans. Biomed. Eng., vol. 44, no. 11, pp. 1114 - 1121, Nov. 1997.

[9] G.W., McLaughlin, "Microfluidic and biosensor applications of fluoropolymer films," in Departement of Electrical Engineering, Stanford University,2001.

[10] P. J. Rousche, et. al., "Flexible polyimide-based intracortical electrode arrays with bioactive capability," IEEE Trans. Biomed. Eng., vol. 48, no. 3, pp. 361-371, Mar. 2001.

[11] C.-S. Kim, et.al, "Manipulation of microenvironment with a built-in electrochemical actuator in proximity of a dissolved oxygen microsensor," IEEE Sensors J., vol. 4, no.5, pp. 568-575, Oct. 2004.

[12] J. Park, C.-S. Kim, S. Zhang and M. Choi, "Glucose oxidase (GOD)coupled amperometric microsensor with integrated electrochemical actuation system," in IEEE Instrum. and Measur. Tech. Conf., May 2005.

[13] C.-C. Wu, T. Yasukawa, H. Shiku, and T. Matsue, "Fabrication of miniature Clark oxygen sensor integrated with microstructure," Sens. Actuators B, Chem., vol. 110, no. 2, pp. 342-349, 2005.

[14] L. Lam, J. Bilek, and J. Atkinson, "Studies on the temperature distribution of a thick film transcutaneous oxygen sensor and its thermal influences on oxygen measurement," IEEE Trans. Biomed. Eng., vol. 53, no. 11, pp. 2341 - 2346, Nov. 2006.
[15] S. Lee, B. L. Ibey, G. L. Coté, and M. V. Pishko, "Measurement of $\mathrm{pH}$ and dissolved oxygen within cell culture media using a hydrogel microarray sensor," Sens. Actuators B, Chem., vol. 128, no. 2, pp. 388398, 2008.

[16] J. Park, Y. K. Pak, and J. J. Pak, "A microfabricated reservoir-type oxygen sensor for measuring the real-time cellular oxygenconsumption rate at various conditions," Sens. Actuators B, Chem., vol. 147, no. 1, pp. 263-269, 2010.

[17] K. J. Cash and H. A. Clark, "Nanosensors and nanomaterials for monitoring glucose in diabetes," Trends in Molecular Medicine, vol. 16, no. 12, pp.584-593, Dec. 2010.

[18] C.-C. Wu, H.-N. Luk, Y.-T. T. Lin, and C.-Y. Yuan, "A Clark-type oxygen chip for in situ estimation of the respiratory activity of adhering cells," Talanta, vol. 81, nos. 1-2, pp. 228-234, 2010.

[19] A. Niazi and C. J. Anthony, "Development of oxygen sensor by integrating the low cost printed circuit board technology and solid electrolyte membrane," in Proc. Int. Conf. Biomed. Eng. Syst., 2014, pp. 1-7.

[20] J. Luo and R. Eitel, "An integrated low temperature co-fired ceramicbased Clark-type oxygen sensor," IEEE Sensors J., vol. 17, no.6, pp. 1590-1595, Mar. 2017.

[21] M. Nosrati, "On the magnetic current density in Maxwell's equations based on Noether theorem," Arxiv: 1911.08880, Physics.Gen-Ph, Nov.2019.

[22] R. Taylor and L. Scribner, eds., The Measurement and Correction of Electrolyte Resistance in Electrochemical Tests. West Conshohocken, PA, USA: ASTM International, 1990.

[23] B. Venema, et. al., "Advances in reflective oxygen saturation monitoring with a novel in-ear sensor system: results of a human hypoxia study," IEEE Trans. Biomed. Eng., vol. 59, no. 7, pp. 2003 2010, Jul. 2012.

[24] S. Bhadra et al., "Fluid Embeddable Coupled Coil Sensor for Wireless $\mathrm{pH}$ Monitoring in a Bioreactor," IEEE Transactions on Instrumentation and Measurement, vol. 63, no. 5, pp. 1337-1346, May 2014.

[25] D. B Papkovsky and R. I Dmitriev, eds., Quenched-phosphorescence Detection of Molecular Oxygen: Applications in Life Sciences. West Croydon, UK: The Royal Society of Chemistry, 2018.

[26] H. Takeuchi, A. Wung, Xin Sun, R. T. Howe and Tsu-Jae King, "Thermal budget limits of quarter-micrometer foundry CMOS for postprocessing MEMS devices," IEEE Transactions on Electron Devices, vol. 52, no. 9, pp. 2081-2086, Sept. 2005.

[27] Y. Huang, A. J. Mason, "Lab-on-CMOS integration of microfluidics and electrochemical sensors," Lab Chip. Vol. 13, no. 9, pp 3929-3934, Oct. 2013.

Mehdi Nosrati received the Ph.D. degree in microwave and electromagnetics from the University of Alberta, Edmonton, $A B$, Canada. As a Post-Doctoral Fellow with the University of Alberta, his research focused on design and fabrication of 3-D waveguide tunable phase shifters for $5 \mathrm{G}$ systems.

He pursued the research working on the design of Nanotechnology-based MEMS sensor for clinical and biomedical applications at Mcgill University, Montreal, QC, Canada. Dr. Nosrati has recently joined to the National Research Council of Canada (NRC) as a Research Associate. His research interests include microwave and millimeter-wave passive devices and circuits, frequencydependent lumped element analysis of distributed-element structures, and microwave sensor design for biomedical applications.

Daniela Vieira, materials engineer, is a $\mathrm{PhD}$ candidate in the Department of Experimental Surgery, McGill University in Montreal, Canada. Her research interests include nanostructured sensors, electrochemistry and nano materials for point-of-care diagnostics.

Edward J. Harvey is a professor of surgery at McGill Universityholding the Michal and Renata Hornstein Chair in Surgical Excellence. $\mathrm{He}$ is leader of the Injury-Repair-Recovery Research Program at the Research Institute of the McGill University Health Centre. He is the founder of sensor based companies looking for medical oriented solutions. Over his career, Harvey has received more than 100 recognitions and 80 grants, and is currently the editor-in-chief of the Canadian Journal of Surgery. 
Geraldine E. Merle, MSc. PhD in Materials Sciences is an Assistant Professor in the Department of Chemical engineering, Polytechnique, Montreal. She holds theTransMedTech Research Chair in Chemical Engineering. Her research interests are to develop low cost and easy to use diagnostic devices and to implement electrochemistry to clinical analysis.

Sharmistha Bhadra received the B.Sc. degree in computer engineering from the University of New Brunswick, Fredericton, NB, Canada, and the M.Sc. and Ph.D. degrees in electrical engineering from the University of Manitoba, Winnipeg, MB, Canada. From 2015 to 2016, she was an NSERC Postdoctoral Fellow with the University of British Columbia, Vancouver, BC, Canada. She joined McGill University in 2016, where she is currently an Assistant Professor. She has published numerous articles and holds two patents in sensor and wearable area. Her current research interests are in the area of printed and flexible hybrid electronics, microelectromechanical systems, wearables and implants and sensors and actuators. 